\title{
Isolation and Characterization of Zinc Solubilizing Bacteria from Kashmir Himalayas, India
}

\author{
Chanrda Sekhar Jagana*, Zahoor Ahmad Baba', S.I. Krishnanand, \\ M.Y. Zargar ${ }^{2}$, Zaffar Badri ${ }^{3}$ and I.J. Khan ${ }^{1}$ \\ ${ }^{1}$ Biofertilizer Research Laboratory, Faculty of Agriculture Wadura \\ ${ }^{2}$ Directorate of Research, Shalimar Campus \\ ${ }^{3}$ Division of Plant Pathology, Faculty of Agriculture Wadura, Sher-e-Kashmir University of \\ Agricultural Sciences and Technology of Kashmir, J\&K, India \\ *Corresponding author:
}

\section{A B S T R A C T}

\begin{tabular}{|l|}
\hline K e y w o r d s \\
Bacillus cereus; \\
Brinjal; \\
Colletotrichum \\
capsici; Zinc \\
solubilizing bacteria \\
\hline Article Info \\
\hline $\begin{array}{l}\text { Accepted: } \\
\text { 12 May } 2019 \\
\text { Available Online: } \\
\text { 10 June } 2019\end{array}$ \\
\hline
\end{tabular}

\section{Introduction}

Zinc micronutrient plays active role in various metabolic processes in plants as well as animals. It is present as co- factor and metal activator in many enzymes. The role of zinc in the nutrition and physiology of both eukaryotic and prokaryotic organisms is widely studied. Such as the maintenance of structural and functional integrity of biological membranes and facilitation of
Rhizosphere soil samples from Brinjal crop were collected from the 12 different locations in north Kashmir districts with the objective to isolate the zinc solubilizing bacteria, their screening and characterization. Zinc carbonate was used as insoluble zinc source. Out of $80 \mathrm{Zn}$ solubilizers, 10 most outstanding isolates were maintained for further screening for mineral solubilization ( $\mathrm{Zn}$ and $\mathrm{K}$ ). Among these $\mathrm{Z}_{\mathrm{n}} \mathrm{sb}(\mathrm{Kup}) \mathrm{M} 2$ which was identified as Bacillus cereus by molecular techniques, showed maximum solubilization index (S.I) and solubilization efficiency (S.E) with 2.4 and $340 \%$ at $48 \mathrm{hrs}$ and 3.8 and $480 \%$ at $72 \mathrm{hr}$ of incubation respectively. In broth containing zinc carbonate, the isolate Bacillus cereus $\left\{Z_{\mathrm{n}} \mathrm{sb}\right.$ (Kup)M2 $\}$ released significantly highest zinc content of $12 \mathrm{ppm} / \mathrm{ml} 10$ days after incubation. Four isolates also showed IAA activity, potassium and phosphate solubilization with highest index of 1.28 and 1.66 shown by Bacillus cereus, further the same isolate also possessed antagonism against Colletotricum capsici. protein synthesis and gene expression. Among all metals, $\mathrm{Zn}$ is needed by the largest number of proteins. Zinc-binding proteins make up nearly $10 \%$ of the proteomes in eukaryotic cells, and $36 \%$ of the eukaryotic $\mathrm{Zn}$-proteins are involved in gene expression (Claudia et al., 2006). Tolerance to environmental stress conditions has a high requirement for $\mathrm{Zn}$ to regulate and maintain the expression of genes needed to protect cells from the detrimental effects of stress 
(Cakmak, 2008). The deficiency of zinc adversely affects the growth, development and yield of crop plants (Cakmak, 2008). The cultivation of crops and soil management practices reduce the large amounts of zinc from the pool of soil (Prasad, 2010). Zinc deficiency is widely prevalent world over, in India more than $50 \%$ of soils are deficient in zinc (Singh et al., 2005). The zinc deficiency has been reported from the agricultural soils of Kashmir valley also (Wani et al., 2013). The zinc deficiency in soils is reflected in crops which ultimately adversely affect the human and animal health who consume the food grains with poor zinc contents. Zinc deficiency is a critical nutritional and health problem in human beings, affecting nearly one third of the world population (Hotz and Brown, 2004). The Copenhagen Consensus concluded that zinc deficiency together with vitamin A deficiency as the top priority global issue and it was also recommended that eliminating zinc deficiency will result in immediate high impacts and high returns for humanity in the developing world.

Majority of the soils are containing high content of total zinc that exists in the unavailable forms in minerals like smithsonite $\left(\mathrm{ZnCO}_{3}\right)$, sphalerite $(\mathrm{ZnS})$, zincite $(\mathrm{ZnO})$, franklinite $\left(\mathrm{ZnFe}_{2} \mathrm{O}_{4}\right)$, wellemite $\left(\mathrm{Zn}_{2} \mathrm{SiO}_{4}\right)$, and hopeite $\left(\mathrm{Zn}_{3} \quad\left(\mathrm{PO}_{4}\right)_{2} .4 \mathrm{H}_{2} \mathrm{O}\right)$ (Lindsay, 1979). It is therefore highly important to devise strategies and utilize the technological interventions for improving the zinc availability by mobilizing the already insoluble soil zinc reserves. Biogeochemically very important numerous interactions occur in soils among various minerals and microbes which in most of the cases aid in unlocking of the otherwise trapped or occluded nutrient metal ions. Solubilization can be performed by a number of mechanisms, the most probable ones include excretion of metabolites such as organic acids, proton extrusion or production of chelating agents, (Sayer and Gadd, 1997).Zinc bearing minerals are also solubilized by an array of soil borne bacterial and fungal isolates with special reference to Bacillus, Pseudomonas, Acinetobacter, Glucanacetobacter, Micrococcus etc (Bapiri et al., 2012). The present research was also conducted to screen some potential zinc solubilizing bacterial isolates for their further use in.

\section{Materials and Methods}

\section{Collection of soil samples}

A total of 36 rhizosphere soil samples were collected from brinjal rhizosphere from 12 locations of three districts (Bandipora, Kupwara, Baramulla) of Kashmir valley (four locations from each district and three samples from each location). After collection, a portion of each sample was immediately transferred to laboratory and stored at $4^{0} \mathrm{C}$ for microbial analysis while as the rest part of soil samples was shade dried and powdered and stored physical and chemical parameters.

\section{Isolation of zinc solubilizing bacteria from collected soil samples}

Serial dilution pour plate technique was used for isolation of zinc solubilizing bacteria and $1 \mathrm{gm}$ of rhizosphere soil from each sample was used for serial dilution. The samples were serially diluted upto $10^{-5}$ dilution factor. The modified Pikovaskya's agar media containing (glucose-10.0 g; ammonium sulphate- $1.0 \mathrm{~g}$; potassium chloride- $0.2 \mathrm{~g}$; dipotassium hydrogen phosphate- $0.2 \mathrm{~g}$; magnesium sulphate $-0.1 \mathrm{~g}$; yeast extract- $0.2 \mathrm{~g}$; distilled water- $1000 \mathrm{ml} ; \mathrm{pH}-7.0$ ) given and $0.1 \%$ insoluble zinc carbonate as zinc source was used for the isolation of zinc solubilizing bacteria (Bapiri et al., 2012). Sterilized medium was poured in to sterilized petri plates under aseptic conditions after 
solidification of medium $0.5 \mathrm{ml}$ of diluted sample suspension from $10^{-5}$ dilution was poured on these plates which were incubated at $28 \pm 2^{0} \mathrm{C}$ for 72 hours in BOD incubator. A total of 80 isolates showed zinc solubilization. Pure cultures of these isolates were obtained by repeated streaking and were preserved for further studies (Bunt and Rovira, 1955).

\section{Characterization of isolated zinc} solubiliziing bacteria

Preliminary characterization of the some outstanding zinc solubilizers was performed on the basis of colony features, morphological characteristics and biochemical tests like Gram's staining, Hydrogen sulphide test, catalase test, starch hydrolysis test, methyl red test, urease test, Voges-Proskauer test, casein hydrolysis test, gelatin liquification, growth at $7 \% \mathrm{NaCl}$, citrate utilization (Archana et al., 2013). By morphological and biochemical characterization the selected isolates were identified up to genus level.

\section{Screening of isolates for qualitative and quantitative zinc solubilization}

For qualitative analysis plate assay was used and for quantitative analysis broth assay was used.

\section{Plate assay}

Isolated test organisms were inoculated on modified Pikovaskaya's media containing zinc carbonate as zinc source $\left(\mathrm{ZnCO}_{3}\right)$ and incubated at $28 \pm 2^{0} \mathrm{C}$ for $72 \mathrm{hrs}$.

After incubation halo zones were observed around colonies which were measured after $48 \mathrm{hrs}$, and $72 \mathrm{hrs}$ of incubation and zinc solubilization index and solubilization efficiency were calculated by using the following formula (Sunitha kumari et al., 2016):S.I = Solubilization Index
S.I = halo zone diameter- colony diameter /colony diameter

Solubilization efficiency was calculated by using formula: S.E $=$ Solubilization Efficiency

S.E $=$ halo zone diameter/ colony diameter $\times$ 100 .

\section{Broth assay}

For quantitative analysis broth assay was used. The modified Pikovaskaya's broth containing $0.1 \%$ insoluble zinc carbonate $\left(\mathrm{ZnCO}_{3}\right)$ as zinc component was prepared and split into $25 \mathrm{ml}$ aliquots in $250 \mathrm{ml}$ Erlenmeyer flasks and sterilized in an autoclave. Then flasks were inoculated with $1 \mathrm{ml}$ suspension of test culture. Experiment was done in triplicates and control was also maintained. The quantity of zinc solubilized was estimated after $7^{\text {th }}$ day and $10^{\text {th }}$ day of incubation. A portion of the inoculated broth /culture was centrifuged at $10000 \mathrm{rpm}$ for 10 minutes, the supernatant was filtered through Whatman no -1 filter paper and diluted 20 times with double distilled water, later on fed to Atomic Adsorption Spectrophotometer (AAS) for estimation of available zinc content (Sarvanan et al., 2003 )

Screening of zinc solubilizing bacteria for other beneficial plant growth promoting properties

The zinc solubilizing bacterial isolates were also screened for the plant growth promoting properties like potassium solubilization (Aleksandrov et al., 1967), phosphate solubilization (Pikovskaya, 1948), antagonistic activity against plant pathogen Colletotricum capsici (collected from division of plant pathology, SKAUST-K,Wadura), indole acetic acid production and ammonia production (Archana et al., 2013). For 
potassium solubilization, Alexandrov medium used containing $0.2 \%$ mica powder as potassium source and for phosphate solubilization, Pikovaskaya's agar medium was used. These two were done in plate assay method and after spot inoculation of cultures measured halo zone diameter, colony diameter and calculated S.I and S.E. For testing antagonistic activity against to plant pathogen colletotricum capsici potato dextrose agar medium used and tested by duel culture technique (Skidmore and Dickinson, 1976). Indole test was done by using medium (Gorden and Weber, 1951) containing peptone- $20.0 \mathrm{~g}$; which was containing sufficient tryptophan, sodium chloride- $5.0 \mathrm{~g}$; Distilled water- $1000 \mathrm{ml}$; and $\mathrm{p}^{\mathrm{H}}-7.4$ and 0.2 $\mathrm{ml}$ of Kovac's reagent was used for $5 \mathrm{ml}$ of test culture medium. Positive test for indole production was formation of cherry red colour ring.

\section{Results and Discussion}

A total of 80 bacterial isolates showed halo zones on modified Pikovaskaya's medium supplemented with $0.1 \%$ zinc carbonate only top 10 best performers were selected based on halo zone diameter and were maintained in nutrient agar slants for further utilization. After morphological and biochemical characterization the isolates were identified up to genus level as out of 10, eight isolates were Bacillus type and 2 belonged to Pseudomonas genus.

The isolates were named $\mathrm{asZ}_{\mathrm{n}} \mathrm{sb}$ (Kup)M2, $\mathrm{Z}_{\mathrm{n}} \mathrm{sb}$ (Band)S1, $\mathrm{Z}_{\mathrm{n}} \mathrm{sb}$ (Band)S1, $\mathrm{Z}_{\mathrm{n}} \mathrm{sb}$ (Band)S23, $\mathrm{Z}_{\mathrm{n}} \mathrm{sb}$ (Band)S24, $\quad \mathrm{Z}_{\mathrm{n}} \mathrm{sb}$ (Band)S6 ${ }_{2}$, $\mathrm{Z}_{\mathrm{n}} \mathrm{Sb}$ (Band)S $7_{2-5}, \quad \mathrm{Z}_{\mathrm{n}} \mathrm{sb}$ (Band)S9 $9_{2-1}$, $\mathrm{Z}_{\mathrm{n}} \mathrm{sb}$ (Band)S9 $9_{2-2}, \mathrm{Z}_{\mathrm{n}} \mathrm{sb}$ (Band)S32 were further characterized on biochemical basis (Table 1) (Plate 1 and 2). Among the screened 10 isolates $\mathrm{Z}_{\mathrm{n}} \mathrm{sb}$ (Kup)M2 was identified as Bacillus cereus MH429978.1 by molecular techniques, showed maximum solubilization index (S.I) and solubilization efficiency (S.E) with $2.4 \& 340 \%$ at $48 \mathrm{hrs}$ and $3.8 \& 480 \%$ at $72 \mathrm{hr}$ of incubation respectively, these were followed by $\mathrm{Z}_{\mathrm{n}} \mathrm{sb}$ (Band)S1 with 1.16 \& $216.6 \%$ at $48 \mathrm{hrs}$ and $1.66 \& 166.6 \%$ at $72 \mathrm{hrs}$ and least S.I and S.E was shown by $Z_{n}$ sb (Band)S24 at 48 hrs and $\mathrm{Z}_{\mathrm{n}} \mathrm{sb}$ (Band)S $9_{2-2}$ at $72 \mathrm{hrs}$ of incubation (Table 2). All 10 isolates significantly increased available zinc in broth containing zinc carbonate at 7 DAI, and 10DAI. Highest solubilization in broth media was shown by isolate $\mathrm{Z}_{\mathrm{n}} \mathrm{sb}$ (Kup)M2(Bacillus cereus MH429978.1) with $12 \mathrm{ppm} / \mathrm{ml}$, followed by $\mathrm{ZnS1}_{1}$ (band) $11.006 \mathrm{ppm} / \mathrm{ml}$ and $\mathrm{ZnS1}$ (band) $10.529 \mathrm{ppm} / \mathrm{ml}$ at $10 \mathrm{DAI}$ (Table $3)$.

\section{Screening of isolates for other beneficial effects}

All the selected isolates were screened for other beneficial effects such as potassium and phosphate solubilization, Indole acetic acid production and antagonistic activity against Colletotricum capsici (Plate 3). Out of 10 only four isolates such as $\mathrm{Z}_{\mathrm{n}} \mathrm{sb}$ (Kup)M2(Bacillus cereus MH429978.1), $\mathrm{Z}_{\mathrm{n}} \mathrm{sb}$ (Band)S1, $\mathrm{Z}_{\mathrm{n}} \mathrm{sb}$ (Band)S1 $1_{1} \mathrm{Z}_{\mathrm{n}} \mathrm{sb}$ (Band)S6 $6_{2}$ showed potassium solubilization with solubilization index of $-1.28,0.66,0.5$ and 0.66 respectively (Table 4) and similarly four isolates $\mathrm{Z}_{\mathrm{n}} \mathrm{sb}(\mathrm{Kup}) \mathrm{M} 2$ (Bacillus cereus MH429978.1), $\quad Z_{n}$ sb(Band)S1, $\mathrm{Z}_{\mathrm{n}} \mathrm{sb}$ (Band)S1 $1, \quad \mathrm{Z}_{\mathrm{n}} \mathrm{sb}$ (Band)S24 showed phosphate solubilization with solubility index of $1.66,0.25,0.5,0.27$ respectively (Table 5).

In addition to mineral solubilization, the isolates were screened for IAA production and antagonistic activity. Out of all four isolates $\mathrm{Z}_{\mathrm{n}} \mathrm{sb}$ (Kup)M2(Bacillus cereus MH429978.1), $\quad Z_{n}$ sb(Band)S6 2 $\mathrm{Z}_{\mathrm{n}} \mathrm{sb}$ (Band)S $7_{2-5}, \quad \mathrm{Z}_{\mathrm{n}} \mathrm{sb}$ (Band)S9 $9_{2-2}$ showed IAA production qualitatively and one isolate $\mathrm{Z}_{\mathrm{n}} \mathrm{sb}$ (Kup)M2 (Bacillus cereus) showed antagonistic activity against to colletotricum capsici. 
Statastical analysis: Statistical analysis was done by using OP STAT software.

\section{Molecular analysis}

Out of all isolates the most efficient strain was molecularly identified up to species level by using $16 \mathrm{~S}$ rRNA sequencing. For the molecular analysis efficient strain $\mathrm{Z}_{\mathrm{n}} \mathrm{sb}$ (Kup)M2 was sent to Agarkar Research Institute, Pune, Maharashtra. The isolate $\mathrm{Z}_{\mathrm{n}} \mathrm{sb}$ (Kup)M2 was identified as Bacillus cereus MH429978.1 by $16 \mathrm{~S}$ rRNA sequencing.

>ZNM2

CAGTCGAGCGAATGGATTAAGAGCTTGCTCTTATGAAGTTAGCGGCGGACGGGTGA GTAACACGTGGGTAACCTGCCCATAAGACTGGGATAACTCCGGGAAACCGGGGCTA ATACCGGATAACATTTTGAACCGCATGGTTCGAAATTGAAAGGCGGCTTCGGCTGTC ACTTATGGATGGACCCGCGTCGCATTAGCTAGTTGGTGAGGTAACGGCTCACCAAGG CAACGATGCGTAGCCGACCTGAGAGGGTGATCGGCCACACTGGGACTGAGACACGG CCCAGACTCCTACGGGAGGCAGCAGTAGGGAATCTTCCGCAATGGACGAAAGTCTG ACGGAGCAACGCCGCGTGAGTGATGAAGGCTTTCGGGTCGTAAAACTCTGTTGTTAG GGAAGAACAAGTGCTAGTTGAATAAGCTGGCACCTTGACGGTACCTAACCAGAAAG CCACGGCTAACTACGTGCCAGCAGCCGCGGTAATACGTAGGTGGCAAGCGTTATCC GGAATTATTGGGCGTAAAGCGCGCGCAGGTGGTTTCTTAAGTCTGATGTGAAAGCCC ACGGCTCAACCGTGGAGGGTCATTGGAAACTGGGAGACTTGAGTGCAGAAGAGGAA AGTGGAATTCCATGTGTAGCGGTGAAATGCGTAGAGATATGGAGGAACACCAGTGG CGAAGGCGACTTTCTGGTCTGTAACTGACACTGAGGCGCGAAAGCGT

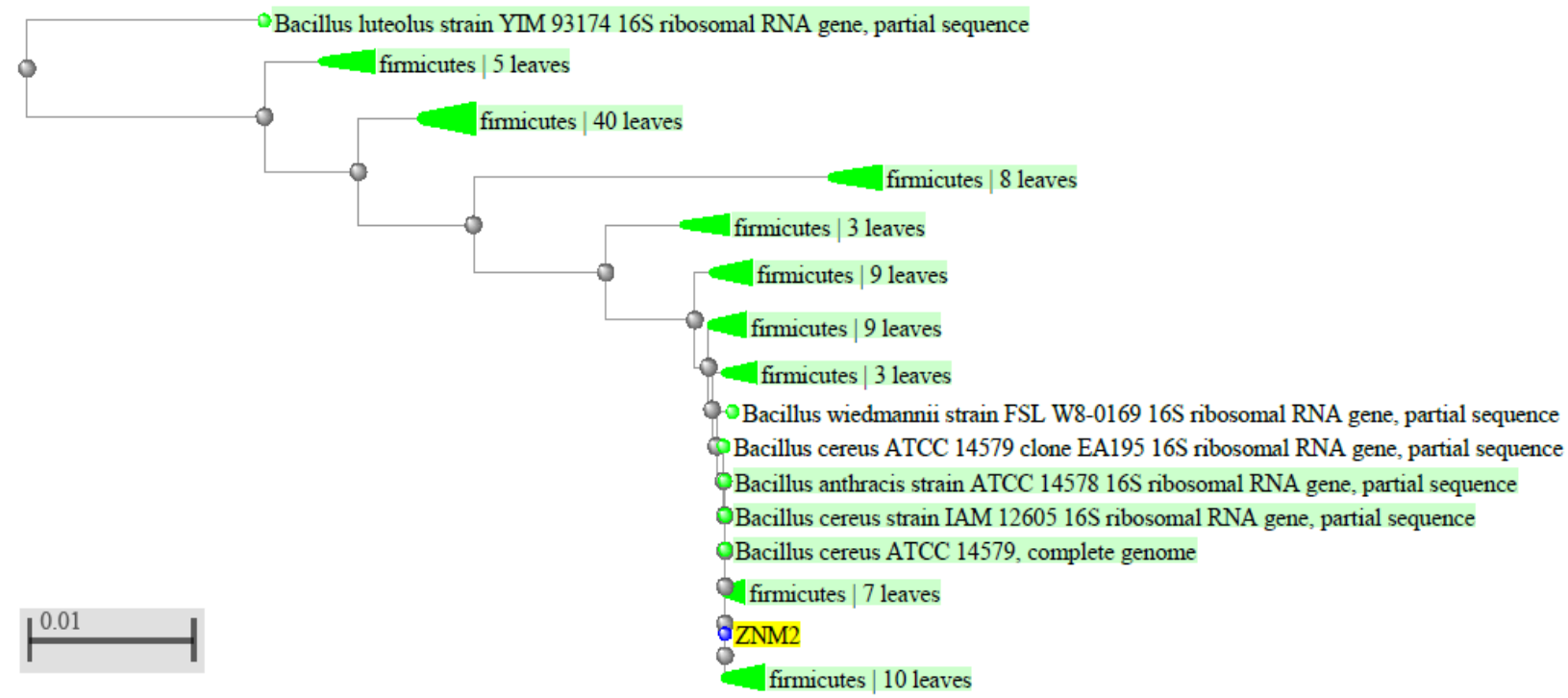

Soil is a habitat for different types of beneficial and harmful microorganisms, which are involved in natural cycles like carbon, nitrogen, phosphorus, potassium and micronutrients. The multiple benefits harvested from this microbial wealth includes improving nutrient availability and controlling plant pathogens besides producing plant growth promoting hormones for better crops. Plants absorb zinc as divalent $\left(\mathrm{Zn}^{+2}\right)$ cation from soil solution. 
Table.1 Morphological and biochemical characters of selected isolates of zinc solubilizing bacteria and probable genus

\begin{tabular}{|c|c|c|c|c|c|c|c|c|c|c|c|c|c|c|c|}
\hline Isolates & $\begin{array}{l}\text { Colony } \\
\text { Characters }\end{array}$ & 1 & 2 & 3 & 4 & 5 & 6 & 7 & 8 & 9 & $\begin{array}{l}\mathbf{1} \\
\mathbf{0}\end{array}$ & $\begin{array}{l}1 \\
1\end{array}$ & $\begin{array}{l}\text { Cell } \\
\text { shape }\end{array}$ & $\begin{array}{l}\text { Gram } \\
\text { reaction }\end{array}$ & Probable Genus \\
\hline $\mathrm{Z}_{\mathrm{n}} \mathrm{sb}(\mathrm{Kup}) \mathrm{M} 2$ & $\begin{array}{l}\text { Yellow, rough, } \\
\text { large, circular, } \\
\text { raise }\end{array}$ & + & + & - & + & + & ++ & + & ++ & + & + & + & Rods & $+\mathrm{ve}$ & Bacillus cereus \\
\hline $\mathrm{Z}_{\mathrm{n}} \mathrm{sb}($ Band)S1 & $\begin{array}{l}\text { smooth, creamy } \\
\text { bluish white, } \\
\text { raise }\end{array}$ & + & - & - & + & + & ++ & + & ++ & - & + & + & $\begin{array}{l}\text { Short } \\
\text { rods }\end{array}$ & -ve & Pseudomonas sp \\
\hline $\mathrm{Z}_{\mathrm{n}} \mathrm{sb}($ Band $) \mathrm{S} 1_{1}$ & $\begin{array}{l}\text { white, rough, } \\
\text { small, flat } \\
\text { surface }\end{array}$ & + & - & ++ & + & - & ++ & + & + & + & + & + & Rod & $+\mathrm{ve}$ & Bacillus sp \\
\hline$Z_{n} s b($ Band $) S 23$ & $\begin{array}{l}\text { White, rough, } \\
\text { circular }\end{array}$ & + & - & ++ & + & + & ++ & + & + & + & + & - & Rod & $+\mathrm{ve}$ & Bacillus sp \\
\hline $\mathrm{Z}_{\mathrm{n}} \mathrm{sb}($ Band)S24 & $\begin{array}{l}\text { White, rough, } \\
\text { circular }\end{array}$ & + & - & ++ & + & - & ++ & + & + & + & + & - & Rod & $+\mathrm{ve}$ & Bacillus sp \\
\hline $\mathrm{Z}_{\mathrm{n}} \mathrm{sb}($ Band $) \mathrm{S6}_{2}$ & $\begin{array}{l}\text { Sky blue, } \\
\text { Smooth raised }\end{array}$ & + & - & + & + & + & - & + & - & - & + & + & $\begin{array}{l}\text { Short } \\
\text { rods }\end{array}$ & -ve & Pseudomonas sp \\
\hline $\mathrm{Z}_{\mathrm{n}} \mathrm{sb}\left(\right.$ Band)S7 $7_{2-5}$ & $\begin{array}{l}\text { Wheat white } \\
\text { color, rough, flat }\end{array}$ & + & - & ++ & + & + & ++ & - & - & - & + & - & Rod & $+\mathrm{ve}$ & Bacillus sp \\
\hline$Z_{n} \operatorname{sb}($ Band $) S 9_{2-1}$ & $\begin{array}{l}\text { Light pinkish, } \\
\text { rough, flat }\end{array}$ & + & - & ++ & + & - & ++ & + & - & + & + & - & Rod & $+\mathrm{ve}$ & Bacillus sp \\
\hline $\mathrm{Z}_{n} \mathbf{s b}($ Band)S9-2 & $\begin{array}{l}\text { White, rough, } \\
\text { flat }\end{array}$ & + & - & ++ & + & + & ++ & + & - & - & + & - & Rod & $+\mathrm{ve}$ & Bacillus sp \\
\hline $\mathrm{Z}_{\mathrm{n}} \mathrm{sb}($ Band $) \mathrm{S3}_{2}$ & $\begin{array}{l}\text { white, rough, } \\
\text { flat }\end{array}$ & + & - & ++ & + & + & ++ & + & + & + & + & - & Rod & $+\mathrm{ve}$ & Bacillus sp \\
\hline
\end{tabular}

Note: Positive: (++); weakly positive (+); Negative: (-)

1. Catalase test, 2.Urease test, 3. Methyl red test, 4. Voges-Proskauer test, 5. Starch hydrolysis, 6. Casein hydrolysis,

7. $\mathrm{H}_{2} \mathrm{~S}$ production, 8. Gelatin liquefaction, 9 . Growth at $7 \% \mathrm{NaCl}, 10$. Dextrose utilization, 11. Citrate utilization.

Table.2 Qualitative estimation of Zinc carbonate solubilization through Solubilization index and Solubilization efficiency

\begin{tabular}{|c|c|c|c|c|c|c|c|c|}
\hline Isolate & & $48 \mathrm{hr}$ & ubati & & & $72 \mathrm{hr}$ & ubatio & \\
\hline & $\begin{array}{l}\text { C.D } \\
(\mathrm{mm})\end{array}$ & $\begin{array}{l}\text { H.D } \\
(\mathrm{mm})\end{array}$ & S.I & $\begin{array}{l}\text { S.E } \\
(\%)\end{array}$ & $\begin{array}{l}\text { C.D } \\
(\mathrm{mm})\end{array}$ & $\begin{array}{l}\text { H.D } \\
(\mathrm{mm})\end{array}$ & S.I & $\begin{array}{l}\text { S.E } \\
(\%)\end{array}$ \\
\hline $\begin{array}{l}\mathbf{Z}_{\mathbf{n}} \mathbf{s b}(\text { Kup) } \mathbf{M} 2 \\
\text { (Bacillus cereus } \quad \text { MH429978.1) }\end{array}$ & 5 & 17 & 2.4 & 340 & 5 & 24 & 3.8 & 480 \\
\hline $\mathrm{Z}_{\mathrm{n}} \mathrm{sb}($ Band)S1 & 6 & 13 & 1.16 & 216.6 & 6 & 16 & 1.66 & 266.6 \\
\hline $\mathrm{Z}_{\mathrm{n}} \mathrm{sb}(\mathrm{Band}) \mathrm{S1} 1_{1}$ & 7 & 10 & 0.42 & 142.8 & 7 & 14 & 1 & 200 \\
\hline $\mathrm{Z}_{\mathrm{n}} \mathrm{sb}($ Band)S23 & 6 & 9 & 0.5 & 150 & 6 & 11 & 0.83 & 183.3 \\
\hline$Z_{n} \operatorname{sb}($ Band)S24 & 5 & 6 & 0.2 & 120 & 5 & 8 & 0.6 & 160 \\
\hline $\mathrm{Z}_{\mathrm{n}} \mathrm{sb}($ Band)S6 & 8 & 13 & 0.62 & 162.5 & 8 & 15 & 0.87 & 187.5 \\
\hline $\mathrm{Z}_{\mathrm{n}} \mathrm{sb}\left(\right.$ Band)S7 $7_{2-5}$ & 7 & 11 & 0.57 & 157.1 & 7 & 12 & 0.71 & 171.4 \\
\hline $\mathrm{Z}_{\mathrm{n}} \mathbf{s b}\left(\right.$ Band)S9 $\mathbf{9}_{2-1}$ & 7 & 11 & 0.57 & 157.1 & 7 & 13 & 0.85 & 185.7 \\
\hline $\mathbf{Z}_{n} \mathbf{s b}\left(\right.$ Band)S9 $9_{2-2}$ & 7 & 10 & 0.42 & 142.8 & 7 & 11 & 0.57 & 157.1 \\
\hline $\mathrm{Z}_{\mathrm{n}} \mathrm{sb}($ Band)S3 & 6 & 11 & 0.83 & 183.3 & 6 & 12 & 1 & 200 \\
\hline
\end{tabular}

C.D = Colony diameter; H.D = Halo zone diameter; S.I = Solubilization index; S.E = Solubilization efficiency 
Table.3 Quantitative estimation of Zinc carbonate solubilization

\begin{tabular}{|c|c|c|}
\hline Isolate & $7 \mathrm{DAI}(\mathrm{ppm} / \mathrm{ml})$ & $10 \mathrm{DAI}(\mathrm{ppm} / \mathrm{ml})$ \\
\hline Control & 0.020 & 0.020 \\
\hline $\begin{array}{l}\mathrm{Z}_{\mathrm{n}} \mathrm{sb}(\text { Kup) M} 2 \\
\text { (Bacillus cereus } \\
\text { MH429978.1) }\end{array}$ & 9.232 & 12.202 \\
\hline $\mathrm{Z}_{\mathrm{n}} \mathrm{sb}($ Band) $\mathrm{S} 1$ & 8.640 & 10.529 \\
\hline $\mathrm{Z}_{\mathrm{n}} \mathrm{sb}(\mathrm{Band}) \mathrm{S} 1_{1}$ & 8.426 & 11.006 \\
\hline $\mathrm{Z}_{\mathrm{n}} \mathrm{sb}($ Band) $\mathrm{S} 23$ & 5.503 & 6.557 \\
\hline $\mathrm{Z}_{\mathrm{n}} \mathrm{sb}(\mathrm{Band}) \mathrm{S} 24$ & 8.890 & 9.453 \\
\hline $\mathrm{Z}_{\mathrm{n}} \mathrm{sb}(\mathrm{Band}) \mathrm{S} 6_{2}$ & 9.771 & 10.005 \\
\hline $\mathrm{Z}_{\mathrm{n}} \mathrm{sb}$ (Band)S $7_{2-5}$ & 5.465 & 6.220 \\
\hline $\mathrm{Z}_{\mathrm{n}} \mathrm{sb}(\mathrm{Band}) \mathrm{S} 9_{2-1}$ & 7.645 & 8.543 \\
\hline $\mathrm{Z}_{\mathrm{n}} \mathrm{sb}(\mathrm{Band}) \mathrm{S} 9_{2-2}$ & 4.922 & 5.254 \\
\hline $\mathrm{Z}_{\mathrm{n}} \mathrm{sb}(\mathrm{Band}) \mathrm{S} 3_{2}$ & 8.222 & 9.224 \\
\hline C. $\mathrm{D}(\mathrm{p} \leq 0.05)$ & 0.035 & 0.077 \\
\hline $\mathrm{SE}(\mathrm{m})$ & 0.012 & 0.026 \\
\hline
\end{tabular}

Table.4 Qualitative estimation of Potassium solubilization

\begin{tabular}{|l|c|c|c|c|}
\hline Isolate & At 72 hrs of incubation & & \\
\hline & C.D (mm) & H.D $(\mathrm{mm})$ & S.I & S.E (\%) \\
\hline $\begin{array}{l}Z_{\mathrm{n}} \mathrm{sb} \text { (Kup)M2(Bacillus } \\
\text { cereus MH429978.1) }\end{array}$ & 7 & 16 & 1.28 & 228.5 \\
\hline $\mathrm{Z}_{\mathrm{n}} \mathrm{sb}$ (Band)S1 & 6 & 10 & 0.66 & 166.6 \\
\hline $\mathrm{Z}_{\mathrm{n}} \mathrm{sb}$ (Band)S1 & & 9 & 0.5 & 150 \\
\hline $\mathrm{Z}_{\mathrm{n}} \mathrm{sb}$ (Band)S6 & 6 & 10 & 0.66 & 166.6 \\
\hline
\end{tabular}

C.D = Culture Diameter; H.D = Halozone Diameter; S.I = Solubilization index; S.E = Solubilization Efficiency

Table.5 Phosphate solubilization of isolates

\begin{tabular}{|l|c|c|c|c|}
\hline Isolate & At 72 hrs of incubation & & \\
\hline & C.D (mm) & H.D (mm) & S.I & S.E (\%) \\
\hline $\begin{array}{l}\mathrm{Z}_{\mathrm{n}} \mathrm{sb} \text { (Kup)M2 } \\
\text { (Bacillus cereus }\end{array}$ & 6 & 16 & 1.66 & 266.6 \\
\hline MH429978.1) & & & & \\
\hline $\mathrm{Z}_{\mathrm{n}} \mathrm{sb}$ (Band)S1 & 8 & 10 & 0.25 & 125 \\
\hline $\mathrm{Z}_{\mathrm{n}} \mathrm{sb}$ (Band)S1 1 & 10 & 15 & 0.5 & 150 \\
\hline $\mathrm{Z}_{\mathrm{n}} \mathrm{sb}$ (Band)S24 & 11 & 14 & 0.27 & 127.7 \\
\hline
\end{tabular}


Plate.1 Zinc solubilization by zinc solubilizing isolates

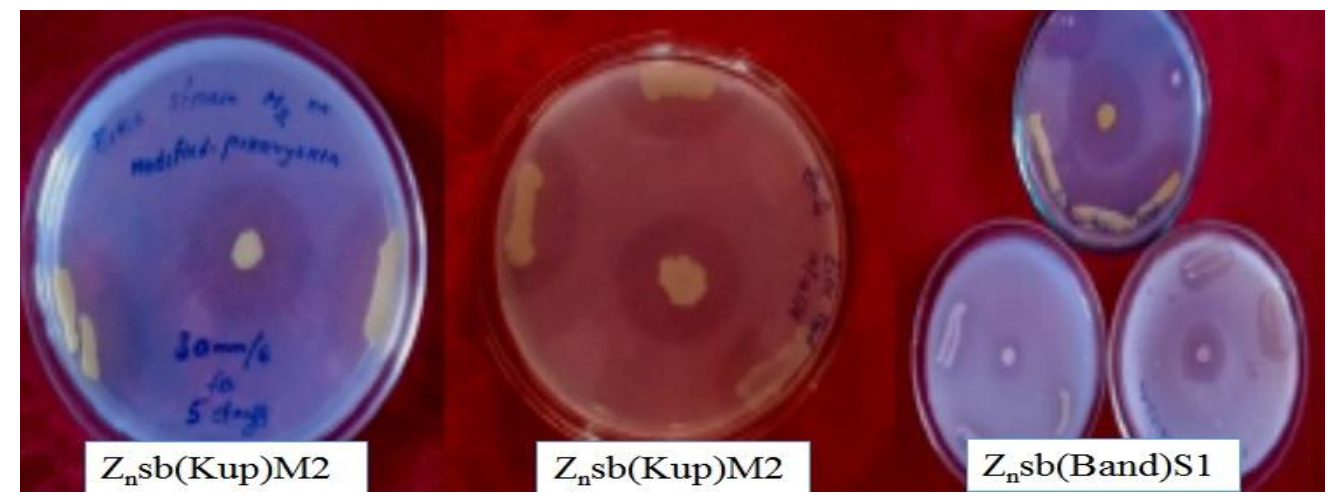

Plate.2 Biochemical tests of zinc solubilizing isolates

a. Catalase activity of zinc solubilizing bacteria
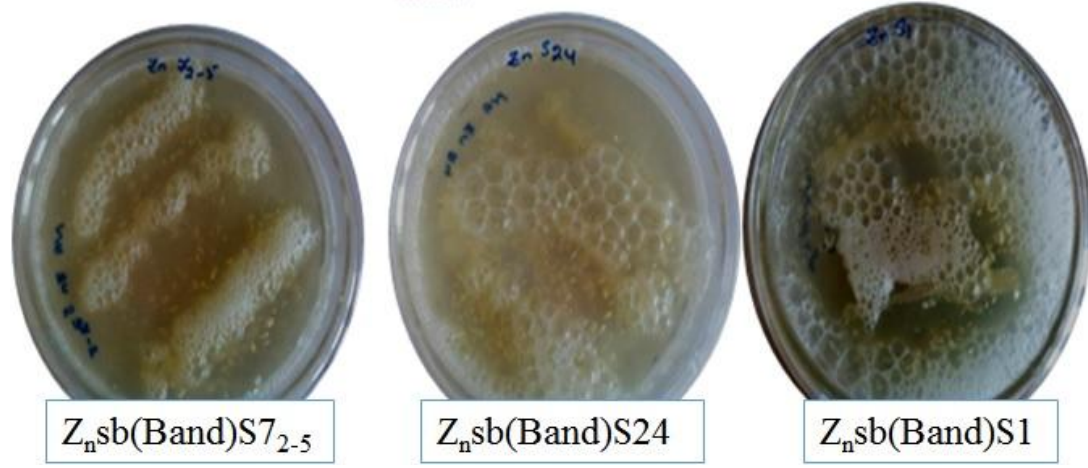

b. Starch hydrolysis activity of zinc solubilizing bacteria

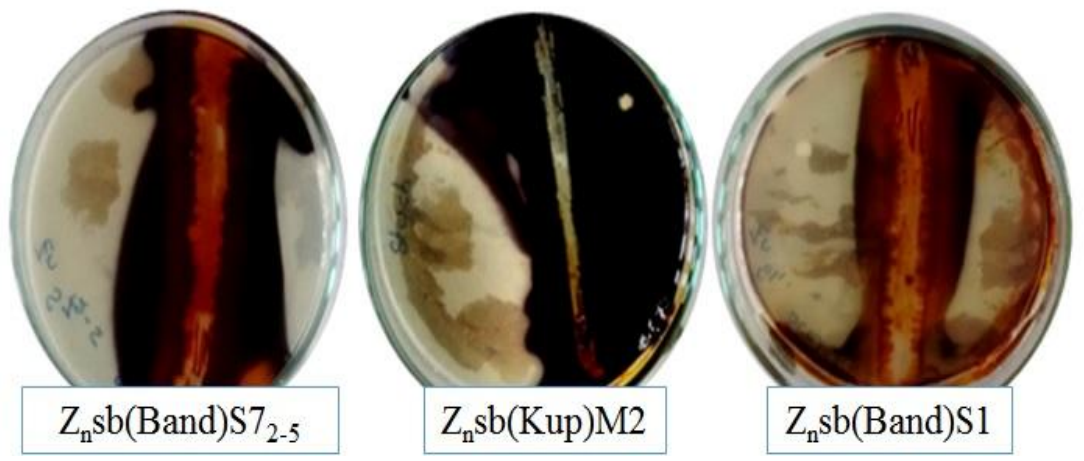




\section{c. Casein hydrolysis activity of zinc solubilizing bacteria}
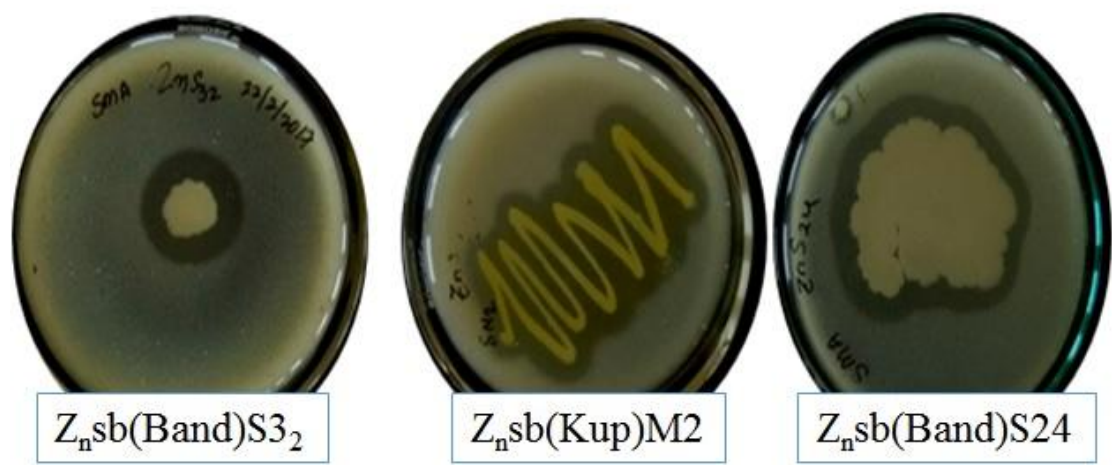

\section{d. Phosphate solubilization of zinc solubilizing bacteria}
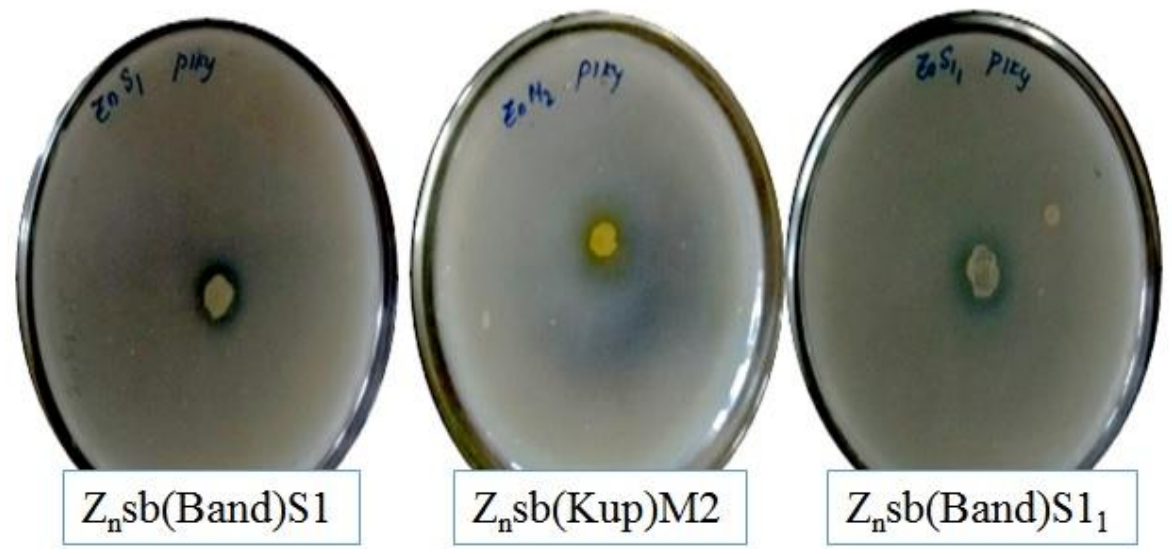

Plate.3 Biocontrol activity of Znsb(Kup)M2(Bacillus cereus MH429978.1) against to colletotricum capsici

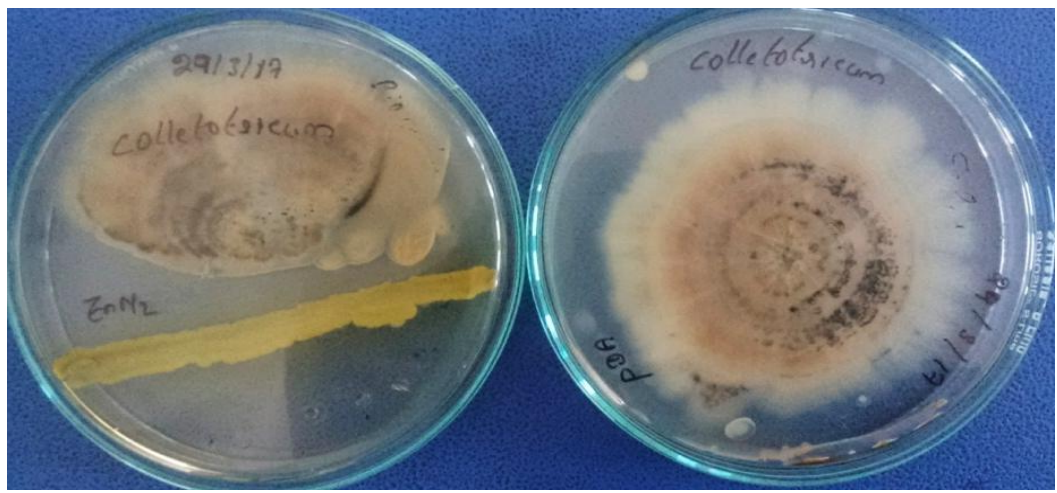

However, major part of the zinc is present in rocks and minerals in unavailable form. It is reported that a variety of microorganisms plays significant role in increasing zinc availability to plants by solubilization of zinc from minerals (Sentil Kumar et al., 2004). Apart from zinc solubilization microbes play important role in solubilization of phosphorus and potassium minerals. Morphologically out of 10, eight isolates were Bacillus type and 2 belonged to Pseudomonas genus and all showed distinct $\mathrm{Zn}$ solubilization efficiency in both qualitatively and quantitatively. These results are supported by the findings of Nazia 
Jamil et al., (2010), Desai et al., (2012), who also isolated Bacillus sp. and Pseudomonas sp. as zinc solubilizers from rhizosphere soils. All isolates showed significant difference in solubilization of mineral $\left(\mathrm{ZnCO}_{3}\right)$ in broth medium. By increasing the incubation period solubilization of zinc mineral also increased quantitatively. Out of all 10 isolates one isolate $\mathrm{Z}_{\mathrm{n}} \mathrm{sb}(\mathrm{Kup}) \mathrm{M} 2$ (Bacillus cereus MH429978.1) showed highest solubilization qualitatively by means of solubilization index (2.4 at $48 \mathrm{hrs}$ and 3.8 at $72 \mathrm{hrs}$ ) and solubilization efficiency (340\% at $48 \mathrm{hrs}$ and $480 \%$ at 72 hrs) followed by $\mathrm{Z}_{\mathrm{n}} \mathrm{sb}$ (Band)S1(Pseudomonas sp.) showed highest S.I (1.16 at 48 hrs and 1.66 at $72 \mathrm{hrs)}$ and S.E (216.6 \% at $48 \mathrm{hrs}$ and $266.6 \%$ at 72 hrs) and so on. Also $Z_{n}$ sb(Kup)M2 (Bacillus cereus MH429978.1) showed highest solubilization of zinc mineral in broth medium with $9.232 \mathrm{ppm} / \mathrm{ml}$ at 7 days after incubation and $12.202 \mathrm{ppm} / \mathrm{ml}$ at 10 days after incubation. These are significantly differentiate with others. These finding were similar to that of earlier findings, who also reported Bacillus cereus and Pseudomonas $s p$. have the ability to good solubilization of zinc minerals (Muhammad shakeel et al., 2015).

The mechanisms of zinc solubilization and other mineral solubilization might be a consequence of proton extrusion, production of organic acids like gluconic acid, oxalic acid, citric acid etc. and other chelating metabolites (Agnihorti, 1970) of microbial origin possibly in a non-specific way leading to solubilization of zinc and other minerals.

\section{References}

Agnihorti, V.P. 1970. Solubilization of insoluble phosphates by some soil fungi isolated from nursery seed beds. Canadian Journal of Microbiology16: 877-880.
Aleksandrov, V.G., Blagodyr, R.N. and Iiiev, I.P. 1967. Liberation of phosphoric apatite by silicate bacteria Mikrobiolohichnyi Zhurnal (Kiev) 29: 111-114.

Archana, D.S., Nandish, M.S., Savalagi, V.P and Alagawadi, A.R. 2013. Characterization of potassium solubilizing bacteria (ksb) from rhizosphere soil. Bioinfolet. 10 (1): 248 - 257.

Azadeh Bapiri., Ahmad Asgharzadeh., Hesam Mujallali., Kazem Khavazi. and Ebrahim Pazira. 2012. Evaluation of Zinc solubilization potential by different strains of Fluorescent Pseudomonads. Journal of Applied Sciences and Environmental Management 16 (3):295298.

Bunt, J.S. and Rovira, A.D., 1955. Microbiological studies of some sub antartic soils. Journal of Soil Science 6:119-128.

Cakmak, I. 2008.Enrichment of cereal grains with zinc: Agronomic or genetic Biofortification. Journal of plant and soil 302(1): 1-17.

Claudia Andreini, Lucia Banci, Ivano Bertini, and Antonio Rosato 2006. Zinc through the Three Domains of LifeJournal Proteome Research 5 (11): 3173-3178.

Desai, S., Praveen Kumar, G., Sultana, U., Pinishetty, S., Ahmed, M. H., Amalraj, D. L. and Gopal Reddy. 2012. Potential microbial candidate strain for management of nutrient require of crops. African Journal of Microbiology Research 6: 3924-3921.

Gorden, A.S and Weber, R.P. 1951. Colorimetric estimation of Indole Acetic Acid. Journal of Plant Physiology 26: 192-195.

Hotz, C., Brown, K.H., 2004. Assessment of the risk of zinc deficiency in populations and options for its control. Food and Nutrtion Buletin 25:94-204. 
Lindsay WL (1979) Chemical equilibrium in soils. Wiley Interscience, New York

Muhammad Shakeel, Afroz Rais Khan, Muhammad Nadeem Hassan and Fauzia Yususf Hafeez, 2015.Root Associated Bacillus sp. Improves Growth, Yield and Zinc Translocation for Basmati Rice (Oryza sativa) Varieties, Frontiers in Microbiology 6: $1-12$.

Nazia, J., Ali, I. and Uzma, I. 2010. Effect of zinc - phosphate - solubilizing bacterial isolates on growth of Vigna radiata. Annals of Microbiology.

Pikovskaya, R.I. 1948. Mobilization of phosphorus in soil connection with the vital activity of some microbial species. Microbiologiya. 17: 362-370.

Prasad, R. 2010. Zinc biofortification of food grains in relation to food security and alleviation of zinc malnutrition, Current Science 98: 1300-1304.

Saravanan, V. S., Subramoniam, S. R., and Anthoni Raj, S., 2003. Assessing invitro solubilisation potential of different zinc solubilisisng bacterial isolates. Brazilian Journal of Microbiology., 34: 121- 125.

Sayer, A. J. and Gadd, M. G. 1997. Solubilization and transformation of insoluble inorganic metal compounds to insoluble metal oxalates by Aspergillus niger. Mycological Research 101: 653661.

Sentil kumar P.S., Geetha, S.A., Savithri, P., Jagadeeswaran, R. and Ragunath, K.P. 2004. Effect of $\mathrm{Zn}$ enriched organic manures and zinc solubilizers application on the yield, curcumin content and nutrient status of soil under turmeric cultivation. Journal of Applied Horticulture 6: 82-86.

Singh, B., Natesan, S. K. A., Singh, B. K. and Usha, K., 2005. Improving zinc efficiency of cereals under zinc deficiency. Current Science. 88(1):3644.

Sunithakumari, K., Padma Devi, S.N and Vasandha, S., 2016. Zinc solubilizing bacterial isolates from the agricultural fields of Coimbatore Current Science 110 (2): 196- 205.

Wani, M. A., Wani, J.A., Bhat, M.A., Kirmani, N.A., Wani, Z.M., Bhat, M.A. and Nazir, S., 2013. Mapping of soil micronutrients in Kashmir agricultural landscape using ordinary kriging and indicator approach. Journal of the Indian Society of Remote Sensing41: 319-29.

\section{How to cite this article:}

Chanrda Sekhar Jagana, Zahoor Ahmad Baba, S.I. Krishnanand, M.Y. Zargar, Zaffar Badri and Khan, I.J. 2019. Isolation and Characterization of Zinc Solubilizing Bacteria from Kashmir Himalayas, India. Int.J.Curr.Microbiol.App.Sci. 8(06): 1248-1258. doi: https://doi.org/10.20546/ijcmas.2019.806.152 